This item was submitted to Loughborough's Research Repository by the author.

Items in Figshare are protected by copyright, with all rights reserved, unless otherwise indicated.

\title{
Are optimal levels of testosterone associated with better cognitive function in healthy older women and men?
}

PLEASE CITE THE PUBLISHED VERSION

http://dx.doi.org/10.1016/j.bbagen.2009.12.009

PUBLISHER

(C) Elsevier B.V.

VERSION

AM (Accepted Manuscript)

LICENCE

CC BY-NC-ND 4.0

\section{REPOSITORY RECORD}

Hogervorst, Eef, Fiona E. Matthews, and Carol Brayne. 2019. "Are Optimal Levels of Testosterone Associated with Better Cognitive Function in Healthy Older Women and Men?”. figshare.

https://hdl.handle.net/2134/15097. 
This item was submitted to Loughborough's Institutional Repository (https://dspace.lboro.ac.uk/) by the author and is made available under the following Creative Commons Licence conditions.

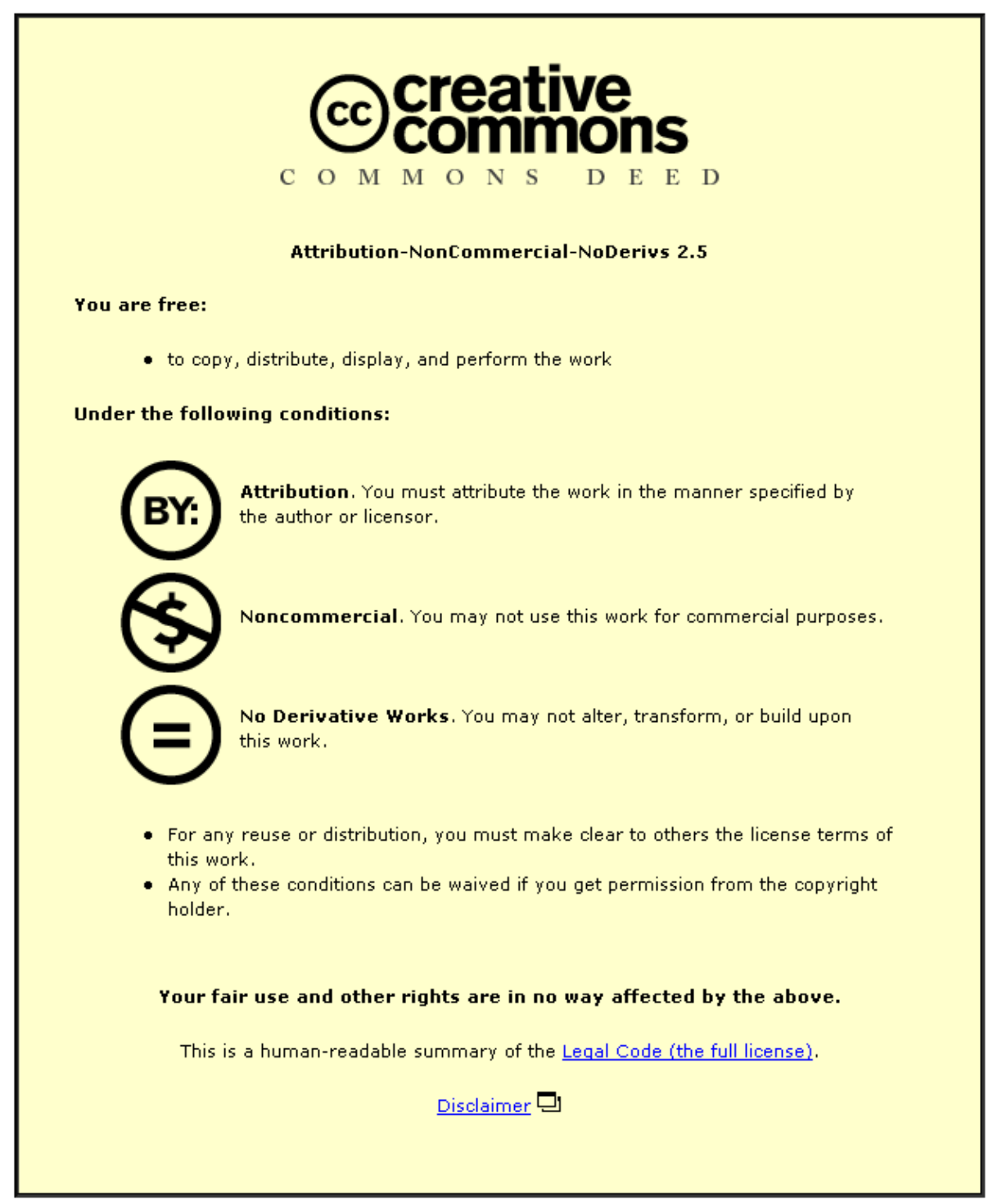

For the full text of this licence, please go to: http://creativecommons.org/licenses/by-nc-nd/2.5/ 
Are optimal levels of testosterone associated with better cognitive function in healthy older women and men ?

Eef Hogervorst, $\mathrm{PhD}^{1}$, Fiona E Matthews ${ }^{2}$, PhD, Carol Brayne, $\mathrm{MD}^{3}$ Department of Human Sciences ${ }^{1}$, Loughborough University, MRC Biostatistics Unit, Institute of Public Health ${ }^{2}$ Department of Public Health and Primary Care ${ }^{3}$, in Cambridge

Address for correspondence

Professor E Hogervorst

Department of Human Sciences

Brockington building

Loughborough LE11 3TU

United Kingdom

Tel + 441509223020

Fax + 441509223940

Email: e.hogervorst@lboro.ac.uk

Acknowledgements:

We would like to acknowledge all staff and participants of the MRC CFAS, MRC Foresight Challenge and OPTIMA, in particular Prof Felicia Huppert and Prof David Smith. This study was supported by the Isaac Newton Trust Cambridge and a Research into Ageing/Help the Aged fellowship. The ESRC funded the Healthy Ageing Study, a sub-study of MRC CFAS. MRC CFAS is funded by the Medical Research Council. FM is supported by MRC.UK.U.1052.00.013. None of the authors has declared any conflicts of interest word count: 6267

Keywords: estrogen, testosterone, sex hormone binding globulin, thyroid hormones, cognition 
Abstract (247 words)

\section{Background}

Sex steroids can positively affect the brain and from this it would follow that high levels of sex steroids could be associated with better cognitive function in older men and women.

\section{Methods}

This Healthy Ageing Study sample comprised of 521 older participants (51\% women) without dementia at baseline, with an age range from 64 to 94 years. Testosterone and sex hormone binding globulin were measured using the automated Immulite 2000 and analyzed in association with baseline memory, global cognitive function and decline (assessed using the Mini-Mental Status Examination or MMSE) and controlling for potential confounds such as age, education, vascular disease, smoking, diabetes, thyroid function, and body mass index.

\section{Results}

In healthy older men and women, optimal levels of testosterone were associated with better MMSE scores at baseline. Follow-up analyses indicated that in men, low testosterone levels $(\mathrm{OR}=.94,95 \% \mathrm{CI}=.88$ to 1.00$)$ were a risk factor for a sharp cognitive decline after two years, perhaps indicative of dementia. Associations were independent of covariates and baseline MMSE. Conversely, women at risk for a sharp drop in cognitive function showed some evidence for higher calculated free testosterone levels at baseline.

\section{Conclusions}

Results replicate earlier cross sectional findings that high levels of sex steroids are not associated with better cognitive function in older people. In men, age accelerated endocrinological change could be associated with dementia pathology.

\section{General Significance}

These data do not support increasing testosterone levels to prevent cognitive decline in men and women over 65 years of age. 


\section{Introduction}

Sex steroids can act on many pathogenic mechanisms thought to be implicated in age-related cognitive decline and dementia and the biological plausibility for estrogens to protect the aging brain has been called 'its strongest suit' ${ }^{1}$. This is probably the main reason why many researchers have continued to investigate sex steroids as a potential prophylactic treatment for dementia and age-related cognitive decline, despite negative publicity surrounding the use of estrogens in the last decade. Large well controlled studies found that estrogen treatment and high estrogen levels increased dementia risk in women over the age of 65 years ${ }^{2-4}$. This may be because older neurons are more likely to undergo pathological change and in this state do not benefit from an estrogenic environment, but instead exhibit acceleration of neuronal degeneration ${ }^{5}$.

Animal and cell culture studies have shown that testosterone can also affect the brain, either indirectly though its conversion into estradiol (the most potent estrogen), but also by acting directly on the brain through androgen receptors (AR). Hippocampal neurons implicated in memory loss in aging and dementia express both estrogen receptors (ER) as well as AR, as do many other brain areas implicated in cognitive function, such as the frontal lobes.

Testosterone's potential protective actions are similar to those of estrogens, such as reduction of oxidative stress and prevention of self programmed cell death. In cell cultures and in vivo animal models testosterone also promoted dendritic sprouting and increased expression of nerve growth factor in the hippocampus, decreased susceptibility to excitotoxicity in hippocampal neurons, reduced amyloid $\beta$-induced neurotoxicity in cultured hippocampal neurons and prevented accumulation of $\beta$-amyloid, and hyperphosphorylation of tau ${ }^{6-10}$, which are all factors considered to be important in the pathogenesis of accelerated pathological age-related cognitive decline, such as Alzheimer's disease (AD), the most common form of dementia. It is unclear whether high testosterone levels are associated with worse cognition in those over 65 years of age, as is thought to perhaps be the case for estrogens ${ }^{11}$. 
Studies investigating endogenous sex steroid levels in association with cognitive function in older women and men have found a wide variety in the direction of results, ranging from positive, negative or no associations ${ }^{11-13}$. Several cross-sectional studies reported positive associations between free testosterone levels (FT, the bioactive fraction not bound to sex hormone binding globulin or SHBG) and cognitive (e.g. memory) functions in older men ${ }^{14-16}$ (but see ${ }^{17}$ who reported non significance of log transformed testosterone in adjusted analyses). In contrast, others found that high FT was associated with lower cognitive function in men of a large age-range (35 to 80 years ${ }^{18,19}$ ), but also that optimal total testosterone (TT) levels (assessed by investigating the quadratic association) did seem to exist for optimal memory performance in older men ${ }^{19,20}$. Longitudinal data, however, did not always confirm significant associations between baseline FT levels with later cognitive decline in men ${ }^{21-23}$, but these often only included one testosterone measurement at baseline. A longitudinal study which used within-individual average (mean) values over repeated evaluations showed that averaged higher FT levels were associated with less visual memory decline in older men, which remained significant in adjusted analyses. While baseline FT also predicted better memory function, a change in TT or FT, however, did not predict cognitive change over time ${ }^{24}$. Traditionally less attention has been given to the association between testosterone levels and cognitive function in women. Testosterone levels in older women are low when compared to those of men. However, because testosterone can be converted into estradiol, relatively small differences in testosterone levels (in nmol/L) could potentially result in large differences in endogenous estradiol levels (in $\mathrm{pmol} / \mathrm{L}$ ). Negative associations were found to exist between memory function and testosterone levels in women ${ }^{12,16}$ but also positive associations were reported in two small studies $(<\mathrm{n}=4025$, ${ }^{26}$ ). There are thus indications that high levels of testosterone, similar to those of estrogens, are not beneficial for maintenance of cognitive function for women over the age of 65 years, while for men perhaps optimal levels exist, which may be additionally age-dependent. We further investigated the associations between testosterone, SHBG, cognition and potential mediators. Unfortunately no funding was available at this stage to include estrogen levels. 


\section{Materials and Methods}

The current sample of the Healthy Ageing Study comprised a subset of respondents originally recruited for the Medical Research Council Cognitive Function and Ageing Study (MRC CFAS). The MRC CFAS is a longitudinal epidemiological study, which is being carried out in six centres in England and Wales (urban Liverpool, Newcastle, Nottingham and Oxford, Cambridgeshire and Gwynedd). Participants from five centre (not including Liverpool) were randomly sampled from the lists of general practitioners in the area and the samples were stratified by age, with equal numbers in the age group 65-74 and 75 and above. Informed consent was obtained by all participants before the onset of the study. Interviews were conducted in the participant's place of residence by trained interviewers. Details of the sampling method and interviews can be found on the website http://www.cfas.ac.uk ${ }^{27,28}$. All respondents were pre-screened for severe cognitive impairment indicative of dementia using i) the MiniMental State Examination (MMSE) score ${ }^{29}$, including only those with a score of 18 or above, and ii) the 'organicity' items from the Automated Geriatric Examination and Computer Assisted Taxonomy (AGECAT) system using the cut-off of ' $\mathrm{O} 3$ and above' as indicative of an organic (dementia) diagnosis ${ }^{30}$. This algorithm using both the MMSE and AGECAT has shown good sensitivity for dementia and depression in older people ${ }^{30}$. For physical frailty, the Townsend disability scale ${ }^{31}$ was employed using a cut-off of 8 , below which participants were not included. Participants also had to have good competence in English and not participate in other MRC CFAS studies.

Participants in this Healthy Ageing Study sub sample comprised of respondents from urban Nottingham drawn from within the city boundaries. The total number who participated in the Healthy Ageing Study (including those from rural Cambridgeshire) was 2041. Sufficient funding was available to obtain blood samples from half of all participants. Individuals willing to provide a blood sample were recruited until at least 500 had been collected in each centre. $76 \%$ of those approached were willing to give a blood sample and were visited at home by a nurse or phlebotomist a median of 6.5 weeks after the Healthy Ageing Study interview. Those 
who were selected and consented to give blood were not substantially different from those who did not in terms of demographics, health, mood and morbidity $\left(\right.$ see $^{32}$ ). At the time of venepuncture in 1992/1993, self report data on health status, medication use and body weight and height (to calculate body mass index or BMI) were recorded. After venepuncture, blood was separated within 4 hours and stored in aliquots at -70 degrees Celsius before samples were assayed for testosterone and SHBG at Loughborough University in 2007.

Testosterone and sex hormone binding globulin (SHBG) were assessed in duplicate $\left(\mathrm{R}^{2}\right.$ $=.97$ for testosterone, $\mathrm{R}^{2}=. .99$ for SHBG) using the automated Immulite 2000, a solid-phase, two- site competitive chemiluminescent immunoassay. The quoted assay range for testosterone is 0.7 to $55 \mathrm{nmol} / \mathrm{L}$, with $0.5 \mathrm{nmol} / \mathrm{L}$ being the limit of detection. For men over 50 years of age, the quoted reference range is 6.28 to $26.7 \mathrm{nmol} / \mathrm{L}$, while for women of this age it is $<0.5$ to 2.57 $\mathrm{nmol} / \mathrm{L}$. Total and between Coefficients of Variation (CV) for the TT assay were reported to be under $15.4 \%$ and were most typically between $4-6 \%$. Using this assay gives a positive bias at concentrations over $2 \mathrm{nmol} / \mathrm{L}^{33}$. For SHBG the between assay CVs were reported to be lower than $8 \%$ and the within assay CVs were lower than $5 \%$, but results were found to be biased up to $29 \%$ depending on the SHBG concentration ${ }^{34}$. Optimal levels were calculated using quartiles for men and women separately.

The Mini-Mental Status Examination (MMSE) ${ }^{29}$ was used as a primary indicator of global cognitive function. A drop of more than 4 points on the MMSE at follow-up (which was an average of 2 years from baseline) was used as an indication of clinically relevant cognitive change possible indicative of a dementia process. In a study of preclinical AD 3 years prior to diagnosis, those who would develop clinical AD had an annual decline of 1.81 points on the MMSE ${ }^{35}$. This would translate to a drop of about 4 points after a 2 year follow-up. As secondary cognitive measures, immediate and delayed recall performance of a news story (Logical Memory, Wechsler Memory Scale-Revised version ${ }^{36}$ ) were used as tests for episodic verbal memory, which had been assessed at baseline only. For both test aspects the maximum 
score is 24. It should be noted that delayed recall was assessed between 5 and 10 minutes after immediate recall, which is shorter than the recommended interval of $30 \mathrm{~min}$.

Health related covariates, which were earlier hypothesized to mediate the association between sex steroid levels and cognitive function, were obtained during the interview and included self reported diagnosed vascular morbidity (risk) factors, such as high blood pressure (BP), coronary heart disease ('heart attack'), stroke, diabetes mellitus, current smoking, body mass index (using weight and height data), and thyroid status using thyroid stimulating hormone (TSH) and free thyroxine (FT4) levels, which had both been assayed within weeks after the interview (see for more details ${ }^{32}$ ). Thyroid disease was assessed by self report and by using blood values with standard laboratory cut-offs ${ }^{32}$

Statistical analyses Descriptive analyses included t-tests (for continuous) and Chi square tests (for categorical data) to describe differences between men and women, as all analyses were stratified for sex (see table 1). Correlations were calculated using Spearman's rank correlations. For cross- sectional data, general linear model analyses were performed using total testosterone (TT, in quartiles calculated for men and women separately) and sex hormone binding globulin levels (SHBG, used in a continuous format, as this variable was normally distributed in men and women) as the main independent variables to assess their association with the following dependent variables: i) global cognitive function, as measured with baseline MMSE performance; ii) verbal memory, as measured by the immediate and delayed news story recall at baseline. For longitudinal data, the delta (change score) of the MMSE (=baseline follow-up score) was used to assess cognitive change over a 2 year period.

A binary variable was computed to perform logistic regression analyses to identify cases with accelerated cognitive decline, who may have been at risk for dementia. For this, a cut-off based on a drop of at least 4 points or more on the MMSE over 2 years was employed. Age at first interview (in years), years of further education, thyroid hormones (both log TSH and FT4 levels, which was normally distributed), and body mass index (calculated using body weight in kg by the square of height in $\mathrm{m}$ ) were entered as covariates. In a second set of 
analyses, the same models were used, but now additionally included self reported diagnosed vascular and other morbidity (stroke, high BP, heart attack, diabetes) and smoking as covariates using a stepwise backward model for all variables ( $<<0.05$ for removal). Analyses were run with and without those with thyroid disease and excluding those who had a baseline MMSE score less than 25. We also substituted the calculated free androgen index (FAI= TT/SHBG x100, which gives a good correlation with physical separation measures in women, but not men ${ }^{37}$ ) in these analyses for TT and SHBG to investigate whether this gave similar results to using TT and SHBG separately. In longitudinal analyses, baseline MMSE scores were also entered. For all analyses SPSS 14.0 was used with a p-value of 0.05 .

\section{Results}

Descriptive analyses of demographics, health and cognitive status

Average age of participants was 74 years of age (SD=6, range 64-94) and $51 \%$ of the sample of 521 participants were women. Participants had received an average of 9.6 (SD 1.7) years of further education. Reflecting Healthy Aging cohort exclusion criteria, only 4\% self reported poor health. Table 1 and Figure 1 show descriptive analyses including self reported morbidity, which was overall low. Twice as many men as women reported having been diagnosed with a heart attack in the past $(\mathrm{p}=0.01)$, but there were no other gender differences in other morbidity (Figure 1) or in current self reported smoking ( $17 \%$ of men and women, $\mathrm{p}=0.80$ ). Most had an MMSE over 25 (table 1) and only $16.3 \%$ of men and $20.6 \%$ of women ( $\mathrm{p}=0.06$ ) complained of memory problems at baseline.

Most participants were in the normal expected range for both TT and SHBG levels. However, only 55\% of men had TT levels over $12 \mathrm{nmol} / \mathrm{L}$ and could thus be classified as eugonadal according to some criteria ${ }^{138}$. Overt hypogonadism was detected in $15.6 \%$ of men who had TT levels lower than $8 \mathrm{nmol} / \mathrm{L}^{38}$, with $6 \%$ having TT levels lower than the detection limit of $0.5 \mathrm{nmol} / \mathrm{L}$.

\footnotetext{
$\overline{{ }^{1} \text { Although criteria for hypogonadism may vary between } \mathrm{TT}}<6$ and $10 \mathrm{nmol} / \mathrm{L}$
} 
More than half (52\%) of women had missing TT data (which were below the .50 detection limit), but 7\% had data over the normal range, with some even reaching the higher end of the range for men (e.g. at 34 and $36 \mathrm{nmol} / \mathrm{L}$, these two were also outside $5 \mathrm{SD}$ from the mean). The assay was done in duplicate producing similar results, so these were not simple typographical errors. The women with these high values were typically quite old (>85 years) and some had thyroid disease, but this would probably not explain such extreme values. Alternatively, fluid contents may have decreased during storage at -70 degrees in a subset of samples due to crystallization and/or insufficient sealing in a minority of tubes, subsequently resulting in an artificial increase in hormone levels. Because we could not further investigate whether these levels were caused by such artefacts or were perhaps due to natural fluctuations with age in the older women, it was decided to calculate separate quartiles for men and women, which would solve the issues regarding the missing data and the data at the higher end of the spectrum for women.

- insert table 1 about here please -

\section{Cross sectional analyses of hormone status and cognitive function at baseline}

Spearman's rank correlations showed that in women, SHBG was not associated with TT $(\mathrm{p}=.56)^{2}$. High SHBG and low FAI (but not TT, $\mathrm{p}=., 20$ ) levels both had significant association with having a lower BMI ( $\mathrm{r}=-.39, \mathrm{p}=0.001, \mathrm{r}=.35, \mathrm{p}<0.001$ respectively). High SHBG levels were also seen in those who were older $(\mathrm{r}=.16, \mathrm{p}<0.01)$. Lower SHBG levels were seen in those diagnosed with diabetes $(\mathrm{z}=-3.16, \mathrm{p}<0.0001)$. There was a trend association between higher TT levels and better MMSE scores ( $\mathrm{r}=-.11, \mathrm{p}=0.08$ ), although the evidence was weak.

In men, low TT was associated with low SHBG levels ( $r=.42, \mathrm{p}<0.0001)$. High TT and $\mathrm{SHBG}^{3}$ levels were both associated with having a lower BMI $(\mathrm{r}=-19, \mathrm{p}<0.002$, respectively $\mathrm{r}=-$ $.24, \mathrm{p}<0.001)$. Having low TT levels was associated with high TSH levels $(\mathrm{r}=-.17, \mathrm{p}<0.0001)$. High FAI (but not SHBG or TT) levels had an inverse association with age ( $r=-15, \mathrm{p}=0.02)$. FAI

\footnotetext{
${ }^{2}$ In women FAI was inversely associated with SHBG, $\mathrm{r}=-.65 \mathrm{p}<0.0001$, and positively with TT levels, $\mathrm{r}=.75, \mathrm{p}<0.0001$

${ }^{3}$ but not FAI, which was associated with both SHBG $r=-38$ and TT $r=.61$, both $p<0.0001$ in men
} 
levels were lower in those diagnosed with stroke (confirmed by M-W U-test: $\mathrm{z}=-2.79, \mathrm{p}<0.005$, with a similar effect seen for TT). An older age was associated with lower MMSE and memory function in men (both $\mathrm{r}=-.21, \mathrm{p}<0.001$ ), and in women (both $\mathrm{r}=-.16, \mathrm{p}<0.01$ ).

- $\quad$ insert fig 1 about here please -

In women, using general linear models, a significant association between optimal TT levels (in quartiles) and MMSE scores at baseline was detected $[\mathrm{F}(1,228)=4.39, \mathrm{p}=0.01]$, with those who had TT levels between 0.50 and $1.09 \mathrm{nmol} / \mathrm{L}$ having the highest MMSE scores (see Figure 2). This was after adjustment for age $[F(1,228)=5.43$, $\mathrm{p}=0.02]$, SHBG $(\mathrm{p}=.39)$, FT4 ( $\mathrm{p}=.33), \log$ transformed TSH levels $(\mathrm{p}=.18), \mathrm{BMI}(\mathrm{p}=.33)$ and education $(\mathrm{p}=.24)$. When vascular (risk) factors were additionally controlled for in separate analyses (with some evidence of a negative association for smoking, $\mathrm{p}=0.06$ ), results remained otherwise unchanged (for TT tertiles, $\mathrm{F}(3,222)=3.10, \mathrm{p}=0.05)$. Analyses (excluding those with thyroid disease, an MMSE score lower than 25 and controlling for age, education, BMI, SHBG and thyroid hormone (TSH and FT4) levels, vascular disease, smoking and diabetes) also showed a significant association for TT tertiles levels ( $\mathrm{p}=0.02$ ). However, there was no significant association between memory or an average drop in MMSE scores over two years with baseline TT, SHBG or FAI levels for women, in or excluding those with thyroid disease, with or without control for covariates. There was some evidence that TT tertiles ( $\mathrm{p}=0.09$ in adjusted analyses) were associated with better performance of the delayed recall. Women in the highest TT quartile recalled $10.46(+/-.63)$ words after a delay, while women in the lowest quartiles recalled 9.2 words (+/-.41).

- insert fig 2 about here please --

In men, using general linear models, no significant associations were found between TT quartiles or SHBG levels and cognition, as measured by baseline memory or MMSE performance, or by using the average drop in MMSE scores over a 2 year follow-up. Only an 
older age $(F(1,245)=20.84, p<0.0001$ resp. $F(1,244)=15.92, p<0.0001)$ was associated with, respectively, a lower baseline MMSE and a lower immediate recall performance. There was some evidence of associations with high log transformed TSH levels $(F(1,245)=2.68, p=0.10$ and $\left.\mathrm{p}=0.07^{4}\right)$. For delayed recall only an older age $(\mathrm{p}<0.0001)$ predicted significantly lower scores. SHBG ( $>>.50)$ and TT ( $>$ >.50) levels did not have significant contributions in these analyses, which were further controlled for FT4 levels, BMI, and education.

When those with thyroid disease and MMSE scores less than 25 were excluded and age, education, SHBG and thyroid hormone (TSH and FT4) levels, BMI, smoking and vascular and other disease (stroke, high BP, heart attack, diabetes) were controlled for in analyses of baseline MMSE, age $(p=0.001)$, education $(p=0.001)$ and some evidence for TT tertiles was seen $(F(3$, 213) $=2.17, \mathrm{p}=0.09$ ), but none of the other variables came into the equation. Post hoc pairwise comparisons (see Figure 3) revealed optimal TT levels for optimal MMSE performance in men, where those who had TT levels in the last quartile (>16.59 nmol/L) also had significantly lower MMSE performance than those of the second $(\mathrm{p}=0.02)$, with similar, trends for the third $(\mathrm{p}=0.06)$ and lowest quartiles $(\mathrm{P}=0.09)$. These results were not seen for memory or the calculated average drop in MMSE scores over time. We subsequently calculated a new binary variable for those who may have been at risk for dementia and had experienced a drop of 4 or more points on the MMSE within the two years of follow-up. insert fig 3 about here please

Analyses of participants who experienced sharp cognitive decline over time versus controls Sex stratified Chi-square analyses of those (30 men, 35 women, including those with thyroid disease) who would show a sharp drop in MMSE performance at follow-up, showed that these cases were at least twice more likely to already report memory problems at baseline (in men: $30 \%$ versus $15 \%$ of controls, $\mathrm{p}=0.04$, in women: $25 \%$ versus $9 \%, \mathrm{p}=0.008$ ). Male cases (but not female cases, $\mathrm{p}=.78$ ) were 10 times more likely to have an MMSE score lower than 25 at baseline $(16.7 \%$ versus $1.6 \%, \mathrm{p}<0.001)$ indicating that the cognitive decline at follow-up was

\footnotetext{
${ }^{4}$ This disappeared when those with thyroid disease were excluded
} 
probably related to an ongoing dementia process, despite screening for this at baseline using the AGECAT algorithm. These analyses revealed no differences between cases and controls in self reported vascular morbidity, for the diagnoses of heart attack, stroke, high blood pressure, diabetes ( $\mathrm{p}$ all $>$.25) or for current smoking at baseline. In female cases, the risk for thyroid disorders was slightly higher than that in controls $(\mathrm{p}=0.003$ ) with $5.6 \%$ of women cases (versus $0 \%$ of controls) having clinical hypothyroidism (either self reported or confirmed by TSH and FT4 levels) and 2.8\% (versus 1\%) of cases having clinical hyperthyroidism ${ }^{5}$.

Analyses of $13 \%$ of men ( $n=26$ versus $n=172$ controls) and $14 \%$ of women ( $n=29$ versus $n=172$ controls) without thyroid disease ${ }^{6}$ who would show a sharp drop in MMSE at follow-up showed that these already also had worse baseline memory (both immediate and delayed recall with an average of around 3.5 words less recalled on both test aspects in cases compared to controls). There were no significant baseline MMSE differences for this selected sample (both cases and controls scored around 27.84). However, at the 2 year follow-up, the average MMSE score was still 27.80 (SD=1.66) in controls, but was down to an average of $21.48(\mathrm{SD}=2.25)$ in cases. There were only small differences in age (male controls were on average 1.66 years younger, and this was 2.53 years on average for female controls) and education (with a 0.70 year of further education more in control men and 0.04 year in women) and also no differences in BMI, diabetes, vascular disease (risk) factors (all p>.38, although there was some association for smoking, $\mathrm{p}=0.07$ ) or either of the thyroid hormones ( $\mathrm{p}>.50$ ).

However, FAI levels were significantly lower in male cases (average 22, SD=14) than those in controls (average 29, SD=14, $\mathrm{p}=0.04$ ) at baseline. There were also weak evidence in the expected direction for TT (average $11 \mathrm{nmol} / \mathrm{L} \mathrm{SD}=8$ for cases versus $13 \mathrm{SD}=6, \mathrm{p}=0.09$ for controls) and SHBG (average $54 \mathrm{nmol} / \mathrm{L} \mathrm{SD}=24$ for cases versus $47 \mathrm{SD}=19, \mathrm{p}=.09$ for controls)

\footnotetext{
${ }^{5}$ No distinction here was made for those who were treated or not as some were over or undertreated with thyroid hormones as laboratory values suggested by comparing these to self reported diagnosed thyroid morbidity $\mathbf{3 2}$. Hogervorst E, Huppert F, Matthews FE, Brayne C. Thyroid function and cognitive decline in the MRC Cognitive Function and Ageing Study. Psychoneuroendocrinology. 2008;33(7):1013-1022..

${ }^{6}$ there were no significant differences in cases ( $\mathrm{n}=30$ men, $\mathrm{n}=35$ women) and controls for TSH and FT4 levels, but as thyroid disease can affect both bioavailable sex steroid levels and cognition, these were excluded from these analyses
} 
levels. In women this was not the case, instead there was some evidence for the reverse (FAI in controls on average was $3(\mathrm{SD} 7)$, for cases on average was $6\left(\mathrm{SD}=11, \mathrm{p}=.06^{7}\right)$.

Multivariable analyses to establish risk factors for sharp cognitive decline Using multivariable logistic regression analyses showed that in men, a sharp drop in MMSE performance of at least 4 points over 2 years (which may have been indicative of dementia) was significantly associated with high SHBG levels $(\mathrm{OR}=1.02,95 \% \mathrm{CI}=1.00$ to $1.05, \mathrm{p}=0.02)$ and independently with low TT levels $(\mathrm{OR}=.93,95 \% \mathrm{CI}=.87$ to $.99, \mathrm{p}=0.03)$ when controlled for age, BMI, education, FT4, log TSH levels and baseline MMSE performance. Additional control for vascular (risk) factors in separate analyses had little effect on the estimates. In similar analyses, the FAI also gave a risk reduction for a sharp cognitive drop (OR=.95, 95\% CI $=.92$ to $.99, \mathrm{p}=0.03$ ), similar to the results of TT models. Entering TT quartiles using simple contrasts in these logistic regression analyses showed that only men who had TT levels in the upper quartile (more than $16.59 \mathrm{nmol} / \mathrm{L}$ ) had significantly less risk for a sharp drop in MMSE performance $(\mathrm{OR}=.27,95 \% \mathrm{CI}=.08$ to $.93, \mathrm{p}=0.04)$, when compared to men who had TT levels equal to or lower than $9.45 \mathrm{nmol} / \mathrm{L}$ (which was the reference value). There was no increase in risk for men who were in the 'gray area' between 12.65 and $9.45 \mathrm{nmol} / \mathrm{L}$. Entering the curvilinear TT term did not give a better fit than the linear term in these analyses. In women, in similar analyses only trend effects remained for high $\log \mathrm{TSH}(\mathrm{OR}=2.21, \mathrm{p}=0.06)$ to predict a sharp drop in cognitive function at follow-up.

Analyses excluding participants with thyroid disease and those with an MMSE below 25 at baseline did not substantially alter results for men with regards to TT (OR=91, 95\% $\mathrm{CI}=.84$ to .99 ), although now SHBG no longer had a significant association with a sharp drop in MMSE scores ( $\mathrm{OR}=1.0295 \% \mathrm{CI}=1.00$ to 1.04$)$. There was some evidence $(\mathrm{p}=0.07)$ for high normal FT4 levels (similar to results of the larger cohort) to confer a 26\% higher risk for a substantial drop in MMSE scores. These analyses were also controlled for age, log TSH and

\footnotetext{
${ }^{7}$ because TT (average $3.5 \mathrm{nmol} / \mathrm{L}, \mathrm{SD}=8$ in cases, average $1.4 \mathrm{nmol} / \mathrm{L}, \mathrm{SD}=4$ in controls, $\mathrm{p}=.16$ ) and SHBG (average $64 \mathrm{nmol} / \mathrm{L}, \mathrm{SD}=33$ versus average $58 \mathrm{nmol} / \mathrm{L}, \mathrm{SD}=28$, $\mathrm{p}=0.30$ ) levels were both non significantly higher in female cases
} 
BMI, which were all non significant and also controlled for further years of education $(\mathrm{OR}=.61$, 95\% CI=.38 to .99) and baseline MMSE (OR=1.63, 95\% CI=1.07 to 2.48$)$ which did contribute significantly to the equation. When in separate analyses vascular risk factors were additionally controlled for, TT (OR=.91, $\mathrm{P}=0.02)$ and SHBG $(\mathrm{OR}=1.03, \mathrm{p}=0.02)$ still significantly contributed to risk of a sharp drop in cognitive function in men, as well as FT4 (OR=1.16, $\mathrm{p}=0.04)$, and smoking $(\mathrm{OR}=2.61, \mathrm{p}=0.05)$. Excluding those with thyroid disease and MMSE scores less than 25 did not alter these analyses. When substituting FAI (OR=.95, 95\% CI=.92 to .99, $\mathrm{p}=0.02$ ) for TT and SHBG, results were not substantially different. With additional control for vascular factors, thyroid hormones (or thyroid disease) and the other covariates, for FAI results also remained unchanged $(\mathrm{OR}=.97,95 \% \mathrm{CI}=.94$ to $1.00, \mathrm{p}<0.05)$ which was the only variable left in the equation to significantly predict cognitive decline using the stepwise backward model. In analyses pertaining to women, only old age was entered as a non significant predictor for a sharp cognitive decline.

\section{Discussion}

This study showed sex differential associations of TT levels with cognition in older people similar to those reported in earlier studies. In older women and men, optimal TT levels were found at baseline to be associated with better global cognitive function, as measured with the MMSE, but not with memory function. Only in men, did high TT and FAI at baseline protect against a sharp drop in cognitive function after a 2 year follow-up. However, men with such high TT levels (>16.59) already had lower baseline MMSE scores and perhaps would thus show a more gradual slope of decline. On the other hand, these analyses were controlled for baseline MMSE scores. Thyroid results of this sample replicated the findings of the larger cohort which included rural Cambridgeshire ${ }^{32}$.

In MRC Challenge, in women of a similar age, high TT levels had a negative association with memory and speed of information processing ${ }^{12}$. We could not replicate significant memory effects in the current Healthy Ageing sample, but differences in the sensitivity of the 
memory test used (as discussed earlier in ${ }^{32}$ ) may at least partly explain this. In addition, in MRC Challenge ${ }^{12}$, estradiol was entered simultaneously in analyses, which was shown to affect the outcome (i.e. without estradiol, associations of verbal memory with TT were no longer significant), but this variable was unfortunately not available for the Healthy Ageing cohort. In MRC Challenge no significant associations of TT with the MMSE was found, but these were reported in larger another cohort of women of a similar age ${ }^{39}$ and power issues may have accounted for finding a lack of significance of hormones on MMSE scores in the MRC Challenge cohort.

In contrast, in the present study, baseline FAI levels were slightly higher in women who were later shown to have a sharp drop in cognitive function over time. These results are reminiscent of those of the OPTIMA cohort, which showed that female AD cases had both higher estrogen and testosterone levels than controls in analyses controlled for a wide variety of potential confounding health-related variables ${ }^{4,11}$. As baseline TT optimal levels for women were within the normal range (between .90 and 1.09), treatment with testosterone, which may increase levels beyond this range, may thus not be advised to sustain cognitive function over time in older women. This would be in line with recent recommendations for estrogen treatment, but may also suggest that testosterone is not an appropriate alternative for estrogens in women over the age of 65 years to prevent cognitive decline ${ }^{11}$. Small treatment trials focusing on cognitive improvement using testosterone were usually done in combination with estrogens in relatively recently menopausal women and were only short term ${ }^{11}$. It is unclear whether long term exposure to higher testosterone levels might confer risk for dementia and accelerated cognitive decline in older women, as may be the case for estrogens. Meta-analyses showed that for both older women with dementia and middle-aged surgically menopausal and/or symptomatic women, positive effects of estrogen treatment did not exceed 4 months ${ }^{40,41}$ and were actually reversed after 1 year of treatment in some studies ${ }^{42}$. Some authors have suggested that high levels of gonadotropins are a more important target for research than sex steroids ${ }^{43-46}$ and there is substantial evidence for this hypothesis. For instance, transgenic mice 
which overexpress luteinizing hormone (LH), but which LH receptors are silenced do not exhibit the memory impairment similar transgenic animals with functional LH receptors show

47. Data shown at the Alzheimer's Association meeting in Madrid 2006 by Voyager pharmaceuticals indicated that leuprolide acetate (which lowers gonadotropin levels such as LH, but thus also (indirectly) estrogens and testosterone levels) improved cognitive function in AD cases. It could be proposed that the reduction of high levels of estrogens and perhaps also testosterone actually led to improvement in these cases rather than (or as well as) the reduction in neurotoxic high levels of LH (see article by Casadeus et al. in this issue).

Significant positive associations between TT levels and MMSE performance at baseline were found in men of the Healthy Ageing sample, but only after control for diabetes, vascular and thyroid morbidity and after excluding participants with an MMSE score lower than $25^{8}$. Earlier cross-sectional findings perhaps using fewer covariates (e.g. not excluding those with thyroid disease, not controlling for a wide range of vascular morbidity) in analyses also did not find significant associations between sex hormone levels and the MMSE performance in men, although significant associations were detected between testosterone levels and other cognitive measures ${ }^{12,24}$. In addition, in the BLSA, a greater increase in risk of Alzheimer's disease with low FT was detected at follow-up ${ }^{49}$. Similarly, in men of the Healthy Ageing sample, high TT and FAI were shown to protect against a sharp drop in cognitive decline, which was possibly indicative of dementia. This was independent of their baseline MMSE performance, SHBG levels, vascular and thyroid disease, smoking and BMI, as well as age and education. These results are also reminiscent of OPTIMA data and other cohorts, where men with Alzheimer's disease had lower levels of TT and FAI than controls, when controlling for multiple confounding factors related to health and morbidity, such as medication use, BMI, alcohol abuse, smoking, and diabetes ${ }^{50}$. On the other hand, in cross sectional analyses of the Massachusetts Male Aging Study (MMAS) ${ }^{51}$ when age and similar covariates were controlled for, TT and FT were no longer significantly associated with cognitive function in men. The age

\footnotetext{
${ }^{8}$ This cut-off is commonly used as a sensitive (but not specific) measure to exclude those with dementia 48. Hogervorst E, Combrinck M, Lapuerta P, Rue J, Swales K, Budge M. The Hopkins Verbal Learning Test and screening for dementia. Dementia. 2002;13(1):13-20.
} 
range of men (from 48 to 80 years) studied in the MMAS was wide and all hormones had been log transformed, which was not deemed necessary in our cohorts, possibly because our participants were a priori selected for relative health and not having dementia at baseline. It is possible that morbidity independently driving hypogonadism ${ }^{38}$ and cognitive decline, such as diabetes, thyroid disease cardiovascular disease, is statistically together better explained by their common denominators: low testosterone and/or high SHBG levels. Sex steroid fluctuations perhaps thus act as 'umbrella variables', capturing the variance associated with multiple morbidity (diabetes, thyroid disease, liver dysfunction, wasting) each individually leading to cognitive decline ${ }^{52}$. Other recent findings suggested that high levels of SHBG in themselves are independently of sex hormone levels associated with dementia risk and could be a marker for biological aging ${ }^{53}$. SHBG also increases with estrogen treatment and is associated with high E2 levels (Serin, 1992], which may suggest why it had an independent association with cognition in the present analyses which did not include estrogens. In the MMSA ${ }^{51}$, estrone was seen to have a negative association with spatial function in adjusted analyses. Other longitudinal cohorts also reported that high estrogen- but not testosterone- levels were a risk factor for dementia or markers of dementia, such as cognitive decline and hippocampal atrophy, in both men and women ${ }^{22,54-56}$. Interestingly, in the brains of men with AD and those in early non clinical stages of dementia, testosterone- but not estradiol- levels were also already seen to be lower than in controls ${ }^{57}$. Differences in assays used, including estrogens in the same analyses and possibly, differences in age range and dementia and health assessment at screening of participants make it difficult to compare studies and arrive at clear conclusions.

However, one theoretical explanatory mechanism which combines the different findings could be the following ${ }^{9}$. Aromatase is responsible for an increased conversion of testosterone into estradiol and there is some evidence that the genetic polymorphism associated with increased expression of aromatase is more frequent in men with $\mathrm{AD}$ than controls ${ }^{58}$. Other genetic polymorphisms have been associated with an acceleration of the age-related decline in testosterone ${ }^{59}$ and were found to occur more frequently in men with $\mathrm{AD}^{60}$. In addition, having 
the APOE epsilon 4 allele, a well established genetic risk factor for $\mathrm{AD}$, was seen to be associated with lower TT, also in control men of the OPTIMA case control cohort who had not (yet?) developed dementia symptoms ${ }^{61}$. It may thus be the case that men at risk for AD have genetic polymorphisms that predispose them to accelerated age-related lowering of TT levels and an increased conversion of TT into estrogen levels. As described in the introduction, neurons undergoing pathological change do not benefit from an estrogenic environment. From this, however, it would follow that treatment with testosterone, which may result in increased conversion to estradiol in the brain in men who are genetically at risk, might not be recommended in older men, similar to recommendations for older women.

Earlier it was hypothesized that thyroid dysregulation may be associated with an increase in SHBG, leading to a subsequent lowering of $\mathrm{FAI}^{52}$, leading to increased levels of gonadotropins which are, as described, potentially neurotoxic. However, in the present cohort no association was found between SHBG and thyroid hormones levels. Hypothyroidism assessed at screening using high TSH levels was, similar to findings of the larger cohort ${ }^{32}$, associated with lower cognitive function in men at baseline and with a sharp cognitive drop in women over time, but this was no longer significant when those with thyroid disease were excluded. High FT4 had an independent association with cognitive decline in men, which remained significant in those without overt thyroid disease, similar to that of other cohorts ${ }^{32}$. Smoking, a known modifiable risk factor ${ }^{62}$, also increased the risk for a sharp cognitive drop by more than a factor 2 in this cohort. However, unlike findings in other cohorts ${ }^{62}$, vascular disease or diabetes had no significant associations with MMSE, which raises doubts about the validity of self-report of morbidity. For instance, those diagnosed with high BP or diabetes did not have significantly lower MMSE scores or a faster drop in this cohort as was found by others ${ }^{62}$. Older people are perhaps often also not aware of harboring these 'silent killers'. For instance, our earlier analyses ${ }^{32}$ showed that half of participants with thyroid disease as assessed by blood values were unaware of having this. 
There are thus several limitations to these analyses. There has been no adjustment in the analysis for the study selection, nor for differential dropout as the study was considered too small to enable this to be undertaken reliably. Furthermore, no extensive medical examination was carried out at baseline and only self-report data were available on diagnosed morbidity and medication use which may be particularly questionable in those suspected of having dementia ${ }^{63}$. Patients with dementia or other memory problems by the very nature of their symptoms may be less reliable in recall and carer confirmation when available is also not always accurate.There was also no formal dementia diagnosis. On the other hand, thyroid status was reviewed in detail in this cohort ${ }^{32}$ and the AGECAT algorithm in earlier studies was shown to be a good predictor of depression and dementia in older people ${ }^{30}$. Second, although FAI is considered to be a good predictor of free and bioavailable testosterone in women, this of course depends on the accuracy and sensitivity of the testosterone and SHBG assays primarily ${ }^{37}$. Studies have shown that the DPG Immulite overestimated values in men (as compared to the gold standard ID/GC Mass Spec) and underestimated values in women. In general, the consensus now is that these types of automated assays should not be used in women or children ${ }^{37}$. Indeed, more than half of the data of women were missing in the current cohort leading to low resolution in the data. When these missing data were ignored, negative associations were found for high levels of SHBG (beta=.23, $\mathrm{p}=0.01$ ) and testosterone (beta=-.19, $\mathrm{p}<0.05$ ) with both MMSE and memory function at baseline, although these did not predict cognitive decline over time. In controlled analyses, only the negative associations of SHBG levels with both delayed recall and MMSE performance remained significant in women. However, these data only reflect those of women with relatively high TT levels and did not take into account the half with undetectable data. This we tried to remedy by using quartiles. However, with potential issues because of long term storage and possible crystallization of fluids perhaps explaining high to very high TT levels in women, data need to be regarded with caution. For future assays, the gold standard GC Mass Spec will be used in our studies. 
In sum, in men and women optimal TT levels were associated with better MMSE performance at baseline. Men who would show a sharp cognitive drop indicative of dementia had low TT levels and higher SHBG levels at baseline. This may be associated with an accelerated decline in TT levels impacting on brain function and/or a sign of increased conversion of TT into estrogens related to particular genetic polymorphisms in men at risk for dementia. More research needs to be done to further investigate this. In women, the reverse was the case, with some evidence for higher FAI in those who would show a sharp drop in cognitive function. Combined data at this point do not suggest that testosterone in older men and women would be a viable treatment to prevent cognitive decline.

\section{List of abbreviations}

$\mathrm{AD}=$ Alzheimer's disease

AGECAT= Automated Geriatric Examination and Computer Assisted Taxonomy

$\mathrm{AR}=$ androgen receptor

BLSA=Baltimore Longitudinal Study of Aging

$\mathrm{BP}=$ blood pressure

BMI=body mass index

$\mathrm{CV}=$ coefficient of variation

ER=estrogen receptor

E2=estradiol

FAI=free androgen index

$\mathrm{FT}=$ free testosterone

FT4= free thyroxine

ID/GC Mass Spec= isotope dilution-gas chromatography mass spectrometry

LH=luteinizing hormone

MMAS= Massachusetts Male Aging Study 
MMSE=Mini Mental Status Examination

MRC CFAS= Medical Research Council Cognitive Function and Ageing Study

OPTIMA= Oxford Project To Investigate Memory and Ageing

$\mathrm{OR}=$ odd ratio

TSH=thyroid stimulating hormone

$\mathrm{TT}=$ Total testosterone

SHBG = sex hormone binding globulin 


\section{References}

1. Barrett-Connor E. Rethinking estrogen and the brain. JAGS. 1998;46:918-920.

2. Shumaker SA, Legault C, Rapp SR, et al. Estrogen plus progestin and the incidence of dementia and mild cognitive impairment in postmenopausal women. JAMA. 2003;289:2651-2662.

3. Shumaker SA, Legault C, Kuller L, al. e. Conjugated equine estrogens and incidence of probable dementia and mild cognitive impairment in postmenopausal women: Women's Health Initiative Memory Study. JAMA. 2004;291(24):2947-2958.

4. Hogervorst E, Barnetson, L., Williams, J., Budge, M., Dowsett, M. \& Smith, A.D. Women with dementia of Alzheimer's type have higher levels of estradiol than healthy controls: prize winning poster. Paper presented at: The 5th Annual Graylyn Conference on Women's health, 1999b; Winston-Salem.

5. Brinton RD. The healthy cell bias of estrogen action. Trends Neurosci.. 2008; 31(10) :529-537.

6. Ahlbom E, Prins GS, Ceccatelli S. Testosterone protects cerebellar granule cells from oxidative stress-induced cell death through a receptor mediated mechanism. Brain Res. 2001;892:255-262.

7. Hammond J, Le Q, Goodyer C, Gelfand M, Trifiro M, LeBlanc A. Testosterone mediated neuroprotection through the androgen receptor in human primary neurons. $J$ Neurochem. 2001;77:1319-1326.

8. Pike CJ. Testosterone attenuates b-amyloid toxicity in cultured hippocampal neurons. Brain Res. 2001;919:160-165.

9. Hogervorst E, Bandelow S, Lehmann DL. Testosterone, Gonadotrophins, \& Genetic Polymorphisms in Men with Alzheimer's disease. In: Hogervorst E, Henderson, V.W., Brinton-Diaz, R.D., Gibbs, R, ed. Hormones, Cognition and the Brain. Cambridge: Cambridge University Press; 2009.

10. Pike CJ, Rosario ER. Testosterone regulates Alzheimer's disease pathogenesis. In: Hogervorst E, Henderson, V.W., Brinton-Diaz, R.D., Gibbs, R, ed. Hormones, Cognition and the Brain. Cambridge: Cambridge University Press; 2009:Chapter 26.

11. Hogervorst E, Bandelow S, Moffat SD. Increasing testosterone levels and effects on cognitive function in elderly men and women. Curr Drugs Targets: CNS Neurol Dis. 2005;4(5):531-540.

12. Hogervorst E, De Jager C, Budge MM, Smith AD. Serum levels of estradiol and testosterone and performance in different cognitive domains in healthy elderly men and women. Psychoneuroendocrinology. 2004;29(3):405-421. 
13. Lunenfeld B, Gooren LJG, Morales A, Morley JE. Textbook of men's health and aging. 2 ed: Informa Health Care; 2007:16.

14. Barrett-Connor E, Goodman-Gruen D, Patay B. Endogenous sex hormones and cognitive function in older men. JCEM. 1999;84(10):3681-3685.

15. Yaffe K, Lui LY, Zmuda J, Cauley J. Sex hormones and cognitive function in older men. JAGS. 2002;50(4):707-712.

16. Thilers PP, Macdonald SW, Herlitz A. The association between endogenous free testosterone and cognitive performance: a population-based study in 35 to 90 year-old men and women. Psychoneuroendocrinology. 2006;31(5):565-576.

17. Fonda SJ, Bertrand R, O'Donnell A, Longcope C, McKinlay JB. Age, hormones, and cognitive functioning among middle-aged and elderly men: cross-sectional evidence from the Massachusetts Male Aging Study. J Gerontol A Biol Sci Med Sci. 2005;60(3):385-390.

18. Yonker JE, Eriksson E, Nilsson LG, Herlitz A. Negative association of testosterone on spatial visualization in 35 to 80 year old men. Cortex. 2006;42(3):376-386.

19. Martin DM, Wittert G, Burns NR, Haren MT, Sugarman R. Testosterone and cognitive function in ageing men: data from the Florey Adelaide Male Ageing Study (FAMAS). Maturitas. 2007;57(2):182-194.

20. Muller M, Aleman A, Grobbee DE, deHaan EH, van der Schouw YT. Endogenous sex hormone levels and cognitive function in aging men: is there an optimal level. JCEM. 2005;90(5):2618-2623.

21. Yaffe K, Barnes D, Lindquist $\mathrm{K}$, et al. Endogenous sex hormone levels and risk of cognitive decline in an older biracial cohort. Neurobiol Aging. 2007;28(2):171-178.

22. Geerlings MI, Strozyk D, Masaki K, et al. Endogenous sex hormones, cognitive decline, and future dementia in old men. Ann Neurol. 2006;60(3):346-355.

23. Lessov-Schlaggar CN, Reed T, Swan GE, et al. Association of sex steroid hormones with brain morphology and cognition in healthy elderly men. Neurology. 2005;65(10):1591-1596.

24. Moffat SD, Zonderman AB, Metter EJ, Blackman MR, Harman SM, Resnick SM. Longitudinal assessment of serum free testosterone concentration predicts memory performance and cognitive status in elderly men. JCEM. 2002;87(11):5001-5007.

25. Wolf OT, Kirschbaum C. Endogenous estradiol and testosterone levels are associated with cognitive performance in older women and men. Horm Behav. 2002;May;41(3):259-266.

26. Drake EB, Henderson, V.W., Stanczyk, F.Z., McCleary, C.A., Brown, W.S., Smith, C.A., Rizzo, A.A., Murdock, G.A., Buckwalter, J.G. Associations between circulating 
sex steroid hormones and cognition in normal elderly women. Neurology. 2000;54:599603.

27. CFAS M. Cognitive function and dementia in six areas of England and Wales: the distribution of MMSE and prevalence of GMS organicity level in the MRC CFA Study. The Medical Research Council Cognitive Function and Ageing Study (MRC CFAS). Psychol Med. 1998;28(2):319-335.

28. Pinto EM, Huppert FA, Morgan K, MRC CFAS, Brayne C. Neutrophil counts, monocyte counts and cardiovascular disease in the elderly. Exp Gerontol 1998;39(4):615-619.

29. Folstein M, Folstein S, McHugh P. 'Mini-mental State': a practical method for grading th cognitive state of patients for the clinician. J Psychiatr Res. 1977;12:189-198.

30. Copeland JR, Dewey ME, Griffiths-Jones HM. A computerized psychiatric diagnostic system and case nomenclature for elderly subjects: GMS and AGECAT. Psychol Med. 1986;16:89-99.

31. Townsend P. Poverty in the United Kingdom. U.K.: Pelican; 1979.

32. Hogervorst E, Huppert F, Matthews FE, Brayne C. Thyroid function and cognitive decline in the MRC Cognitive Function and Ageing Study. Psychoneuroendocrinology. 2008;33(7):1013-1022.

33. Lamph S, Wheeler M, Halloran S. DPC Immulite 2000 and immulite testosterone assays. Surrey: MHRA Evaluation Unit Royal Surrey County Hospital Trust; 2004.

34. Jin M, Wener MH, Bankson DD. Evaluation of automated SHBG immunoassays. Clin Biochem. 2006;39(1):91-94.

35. Jones S, Small BJ, Fraiglioni L, Backman L. Predictors of cognitive change from preclinical to clinical Alzheimer's disease. Brain Cognition. 2002;49(2):210-213.

36. Wechsler D. Manual for the Wechsler Memory Scale Revised. San Antonio TX USA: The Psychological Corporation; 1987.

37. Rosner W, Auchus RJ, Azziz R, Sluss PM, Raff H. Position statement: utility, limitations and pitfalls in measuring testosterone: an endocrine society position statement. JCEM. 2007;92(2):405-413.

38. Arver S, Lehtihet M. Current guidelines for the diagnosis of testosterone deficiency. Front Horm Res. 2009;37:5-20.

39. Barrett-Connor E, Goodman-Gruen, D. Cognitive function and endogenous sex hormones in older women. JAGS. 1999;47(11):1289-1293.

40. Yesufu A, Bandelow S, Hogervorst E. Meta-analyses of the effect of hormone treatment on cognitive function in postmenopausal women. Women's Health. 2007;3(2):173-195. 
41. Hogervorst E, Yaffe K, Richards M, Huppert FA. HRT to maintain cognitive function in women with dementia. Cochrane Database Syst Rev. 2009;21(1):CD003799.

42. Hogervorst E, Bandelow S. Should surgically menopausal women be treated with estrogens to decrease the risk for dementia ? Invited Editorial. Neurology. 2007;69(11):1070-1071.

43. Bowen RL, Isley JP, Atkinson RL. An association of elevated serum gonadotropin concentrations and Alzheimer Disease ? J Neuroendocrinol. 2000;Apr;12(4):351-354.

44. Bowen RL, Smith MA, Harris PL, et al. Elevated luteinizing hormone expression colocalizes with neurons vulnerable to Alzheimer's disease pathology. J Neurosci Res. 2002;70(3):514-518.

45. Bowen RL, Verdile G, Liu T, et al. Luteinizing hormone, a reproductive regulator that modulates the processing of amyloid-beta precursor protein and amyloid-beta deposition. J Biol Chem. 2004;279(19):20539-20545.

46. Casadesus G, Atwood CS, Zhu X, et al. Evidence for the role of gonadotropin hormones in the development of Alzheimer disease. Cell Mol Life Sci. 2005;Feb;62(3):293-298.

47. Casadesus G, Milliken EL, Webber KM, et al. Increases in LH are associated with declines in cognitive performance. Moll Cell Endocrinol. 2007;169(1-2):107-111.

48. Hogervorst E, Combrinck M, Lapuerta P, Rue J, Swales K, Budge M. The Hopkins Verbal Learning Test and screening for dementia. Dementia. 2002;13(1):13-20.

49. Moffat SD, Zonderman AB, Metter EJ, et al. Free testosterone and risk for Alzheimer disease in older men. Neurology. 2004;62(2):188-193.

50. Hogervorst E, Williams, J., Budge, M., Barnetson, L., Combrinck, M., \& Smith, A.D. Serum total testosterone is lower in men with Alzheimer's disease. Neuroendocrinol Lett.. 2001;22(3):163-168.

51. Fonda SJ, Bertrand R, O'Donnell A, Longcope C, McKinlay JB. Age, hormones and cognitive function among middle-aged and elderly men. J Gerontol A Biol Sci Med Sci. 2006;60:385-390.

52. Hogervorst E, Bandelow, S., Combrinck MC, Smith AD. Low free testosterone is an independent risk factor for AD. Exp Gerontol. 2004;39(11-12):1633-9

53. Muller M, Schupf N, Manly JJ, Mayeux R, Luchsinger JA. SHBG and incident AD in elderly men and women. Neurobiology Aging. 2008;Nov 18(Epub ahead of print).

54. Ravaglia G, Forti P, Maiolo F, et al. Endogenous sex hormones as risk factors for dementia in elderly men and women. J Gerontol A Biol Sci Med Sci. 2007;62(9):10351041. 
55. Den Heijer T, Geerlings MI, Hofman A, et al. Higher estrogen levels are not associated with larger hippocampi and better memory performance. Arch Neurol. 2003;60(2):213220.

56. Muller M, van den Beld AW, Grobbee DE, de Jong FH, Lamberts SW. Sex hormones and cognitive decline in elderly men. Psychoneuroendocrinology. 2009;34(1):27-31.

57. Rosario ER, Chang L, Stanczyk FZ, Pike CJ. Age-related testosterone depletion and the development of Alzheimer disease. JAMA. 2004;Sep 22;292(12):1431-1432.

58. Iivonen S, Corder E, Lehtovirta M, et al. Polymorphisms in the CYP19 gene confer increased risk for Alzheimer disease. Neurology. 2004;62(7):1170-1176.

59. Krithivas L, Yurgalevitch SM, Mohr BA, et al. Evidence that the CAG repeat in the AR gene is associated with the age-related decline in serum androgen levels in men. $J$ Endocrin. 1999;162(1):137-142.

60. Lehmann DJ, Hogervorst E, Warden DR, Smith AD, Butler HT, Ragoussis J. The androgen receptor CAG repeat and serum testosterone in the risk of Alzheimer's disease in men. JNNP. 2004;75(1):163-164.

61. Hogervorst E, Lehmann DJ, Warden DR, McBroom J, Smith AD. Apolipoprotein E epsilon4 and testosterone interact in the risk of Alzheimer's disease in men. Int $J$ Geriatr Psychiatry. 2002;17(10):938-940.

62. Peters R. The prevention of dementia. Int J Geriatr Psychiatry. 2009;24(5):452-458.

63. Petitti DB, Buckwalter JG, Crooks VC, Chiu V. Prevalence of dementia in users of hormone replacement therapy as defined by prescription data. J Gerontol A Biol Sci Med Sci. 2002;Aug;57(8):M532-538. 